\title{
Freeware for GIS and Remote Sensing
}

\author{
Lena Halounová \\ Department of Mapping and Cartography, Faculty of Civil Engineering \\ Czech Technical University in Prague \\ halounova fsv.cvut.cz
}

Keywords: GIS, Freeware

\begin{abstract}
Education in remote sensing and GIS is based on software utilization. The software needs to be installed in computer rooms with a certain number of licenses. The commercial software equipment is therefore financially demanding and not only for universities, but especially for students. Internet research brings a long list of free software of various capabilities. The paper shows a present state of GIS, image processing and remote sensing free software.
\end{abstract}

\section{Introduction}

GIS and remote sensing tasks are mutually interlinked and division of software to GIS software and remote sensing software means that GIS software is able to work with remote sensing data and to perform simple tasks with them. On the contrary, GIS functions are embedded in remote sensing software. This duality is a result of close relation between these regions. Looking for the GIS free software, three groups of them can be found

- Viewers of commercial software - ArcReader $9^{1}$, ArcExplorer $4.0^{2}$ of ESRI, GeoMedia@ Viewer of INTERGRAPH, FreeLook $3.1^{3}$ for ENVI, etc.

- Software tools for certain tasks as GDAL 1.1.5 [11], libgeotiff, MB-System ${ }^{4}$ for bathymetry and backscatter imagery data derived from multibeam, interferometry, and sidescan sonars, MITAB ${ }^{5}$ MITAB a $\mathrm{C}++$ library for reading and writing MapInfo .TAB (binary) and .MIF/MID files.

- Complete GIS software - GRASS, ILWIS, FMaps covering GIS together with remote sensing.

\footnotetext{
${ }^{1}$ http: //www . arcdata.cz/download/ArcReader/ArcReaderWebSetup. exe

${ }^{2}$ http: //www . arcdata.cz/download/ArcExplorer/AE4JavaSetup. exe

${ }^{3}$ http: //www. envi-sw. com/

${ }^{4}$ http: //www. Ideo. columbia.edu/MB-System/

${ }^{5}$ http://mitab.maptools.org/
} 
- Software for image processing - Intel, Image Analyzer 1.27

The Open Source GIS [7] brings a list of more than 150 free GIS software available for users. The web page remotesensing.org [15] is a source of many free software for remote sensing including the link to Remote Sensing Tutorial prepared by William J. Campbell from NASA [10].

\section{Viewers of commercial software}

Following examples shows limits of viewers for common users.

GeoMedia extregistered Viewer [13]. Key Features of the software are data access providing read-only access to geospatial data in Microsoft Access, MapInfo and Shapefile formats. With GeoMedia Viewer new connections to the above formats can be also created if you have other data sets you want to access. GeoMedia Viewer let us create new geoworkspaces and save any changes made to existing geoworkspaces. GeoMedia Viewer includes a powerful set of navigation commands for moving around and exploring your data as Pan, Zooming, etc. and supports multiple windows including map and data windows.

ArcExplorer [9]. As a complete data explorer, ArcExplorer - Java lets display and query a wide variety of standard data sources. Using ArcExplorer as a stand-alone desktop application, it is possible to consume shapefiles, a variety of images, ArcSDE layers, and more, allows to also pan and zoom through these map layers and identify, locate, and query their spatial and attribute information. You can buffer a selected set of features and view their attributes. ArcExplorer also provides the ability to thematically map your data, symbolizing various features based upon information found in the data's attribute table, displaying graduated symbology, or classbreaks rendering for instance.

\section{Software tools for certain tasks}

There are many software packages for large number of different tasks and questions for GIS purposes [6]. Project conversion software form an important group of them.

GDAL 1.1.5 [11] is a translator library for raster geospatial data formats. As a library, it presents a single abstract data model to the calling application for all supported formats. A initial skeleton of GDAL has formed, and operates for a few formats [11].

Geotiff libs [16] - GeoTIFF represents an effort by over 160 different remote sensing, GIS, cartographic, and surveying related companies and organizations to establish a $\mathrm{TIFF}^{6}$ based interchange format for georeferenced raster imagery [16].

PROJ 4.4.3 [8] - This package offers command line tools and a library for performing respective forward and inverse transformation of cartographic data to or from cartesian data with a wide range of selectable projection functions. O.S. GNU/Linux and other Unices Windows $[8]$.

\footnotetext{
${ }^{6}$ http: //www. awaresystems.be/imaging/tiff/faq.html
} 
Virtual Terrain Project [17] - The goal of VTP is to foster the creation of tools for easily constructing any part of the real world in interactive, 3D digital form. This goal will require a synergetic convergence of the fields of CAD, GIS, visual simulation, surveying and remote sensing. VTP gathers information and tracks progress in areas such as procedural scene construction, feature extraction, and rendering algorithms. VTP writes and supports a set of software tools, including an interactive runtime environment (VTP Enviro). The tools and their source code are freely shared ${ }^{7}$ to help accelerate the adoption and development of the necessary technologies [15].

\section{Complete GIS/remote sensing software}

There are four useful GIS and remote sensing free software packages - GRASS, ILWIS, OSSIM and FMaps.

GRASS - Geographic Resources Analysis Support System commonly referred to as GRASS, this is a Geographic Information System (GIS) used for geospatial data management and analysis, image processing, graphics/maps production, spatial modeling, and visualization. GRASS is currently used in academic and commercial settings around the world, as well as by many governmental agencies and environmental consulting companies. GRASS is a raster GIS. There are a large range of applications of GRASS: geography, landscape ecology, epidemiology, remote sensing ${ }^{8}$, urban planning, biology, geophysics, hydrology, groundwater Flow Modeling (GRASS and MODFLOW ${ }^{9}$ ), vector network analysis (in part of GRASS $6^{10}$ ), geostatistics $^{11}$, raster 3D Volume ${ }^{12}$ (voxel) [5].

ILWIS [1] version 3.4. is a software created for Windows platform and the following list shows the product tools:

- Integrated raster and vector design

- Import and export of widely used data formats

- On-screen and tablet digitizing

- Comprehensive set of image processing tools

- Orthophoto, image georeferencing, transformation and mosaicing

- Advanced modeling and spatial data analysis

- 3D visualization with interactive editing for optimal view findings

- Rich projection and coordinate system library

- Geo-statistical analyses, with Kriging for improved interpolation

- Production and visualization of stereo image pairs

\footnotetext{
${ }^{7}$ http://www.vterrain.org/Site/privacy.html\#License

${ }^{8}$ http://mpa.itc.it/rs/

${ }^{9}$ http://www.valledemexico.ambitiouslemon.com/gwmodelling.html

${ }^{10}$ http://grass.itc.it/grass61/screenshots/network.php

${ }^{11}$ http://grass.itc.it/statsgrass/index.php

${ }^{12}$ http://grass.itc.it/grid3d/
} 
- Spatial Multiple Criteria Evaluation

$52^{\circ}$ North Initiative for Geospatial Open Source Software GmbH is an international research and development company whose mission is to promote the conception, development and application of free open source geo-software for research, education, training and practical use. $52^{\circ}$ North backs an open initiative, which is driven by leading research organizations and individuals in the international GIS field. The work of all partners results in a collection of Java based web services implementations [1].

FMaps [5] is an open source GIS/RS (Geographic Information System/ Remote Sensing) application on the Linux ${ }^{13}$ and Gnome $^{14}$ compatible platforms. The database engine is PostgreSQL ${ }^{15}$. PostgreSQL is an opensource SQL server.

OSSIM (Open Source Software Image Map) [14] is a high performance software system for remote sensing, image processing, geographical information systems and photogrammetry. It is an open source software project maintained at http://www.ossim.org and has been under active development since 1996. The lead developers for the project have years of experience in commercial and government remote sensing systems and applications. OSSIM has been funded by several US government agencies in the intelligence and defense community and the technology is currently deployed in research and operational sites. The name OSSIM is a contrived acronym (Open Source Software Image Map) that is pronounced "awesome" the acronym was established by our first government customer. Designed as a series of high performance software libraries it is written in $\mathrm{C}++$ employing the latest techniques in object oriented software design. A number of command line utilities, GUI tools and applications, and integrated systems have been implemented with the baseline. Many of those tools and applications are included with the software releases.

MultiSpec is a freeware multispectral image data analysis system (latest release: 5-12-2007) MultiSpec is being developed at Purdue University ${ }^{16}$, West Lafayette ${ }^{17}$, IN ${ }^{18}$, by David Landgrebe $^{19}$ and Larry Biehl ${ }^{20}$ from the School of Electrical and Computer Engineering ${ }^{21}$, ITaP $^{22}$ and LARS ${ }^{23}$. It results from an on-going multiyear research effort which is intended to define robust and fundamentally based technology for analyzing multispectral and hyperspectral image data, and to transfer this technology to the user community in as rapid a manner as possible. The results of the research are implemented into MultiSpec and made available to the user community via the download pages. MultiSpec(C) with its documentation(C) is distributed without charge.

\footnotetext{
${ }^{13}$ http://www.linux.org/

${ }^{14}$ http: //www.gnome.org/

${ }^{15}$ http://www.postgresql.org/

${ }^{16}$ http://www.purdue.edu/

${ }^{17}$ http://www. wintek.com/wlaf/

${ }^{18}$ http://www. state.in.us/

${ }^{19}$ http://dynamo.ecn . purdue.edu/ landgreb/

${ }^{20}$ http://dynamo.ecn. purdue.edu/ biehl/

${ }^{21}$ http://www.purdue.edu/ECE

${ }^{22}$ http://www.itap.purdue.edu/

${ }^{23}$ http://www. lars.purdue.edu/
} 


\section{Radar interferometry}

Interferometry is a separate part of remote sensing working with radar image pairs for determination of DEM on one side, and small surface movements - subsidences, e.g., on the other side. It is a separate software and branch as well because its processing method differs significantly from other remote sensing image data evaluation. There is one freeware called DORIS [11].

DORIS (Delft object-oriented radar interferometric software) The Delft Institute of Earth Observation and Space Systems ${ }^{24}$ of Delft University of Technology ${ }^{25}$ developed processor for interferometric SAR. It is a freeware for non-commercial usage. The software works with European ERS and ENVISAT data, Japanese JERS, and Canadian RADARSAT.

\section{Image processing software}

\section{Intel offers Open Source Computer Vision Library with following}

Library areas [12]:

- Image functions: Creation, allocation, destruction of images. Fast pixel access macros.

- Data Structures: Static types and dynamic storage

- Contour Processing: Finding, displaying, manipulation, and simplification of image contours

- Geometry: Line and ellipse fitting. Convex hull. Contour analysis

- Features: 1st \& 2nd Image Derivatives. Lines: Canny, Hough. Corners: Finding, tracking.

- Image Statistics: In region of interest: Count, Mean, STD, Min, Max, Norm, Moments, $\mathrm{Hu}$ Moments

- Image Pyramids: Power of 2. Color/texture segmentation

- Morphology: Erode, dilate, open, close. Gradient, top-hat, black-hat

- and many others

Intel extregistered Image Processing Library (included in OpenCV WinOS download) comprises [12]:

- Image creation and access

- Image arithmetic and logic operations

- Image filtering

- Linear image transformation

- Image morphology

${ }^{24}$ http: //WwW.deos.tudelft.nl/

${ }^{25}$ http: //www . tudelft.nl/live/pagina.jsp?id=b226846d-f19f-4c34-97ed-165fecc5ad8f\&lang=nl 
- Color space conversion

- Image histogram and thresholding

- Geometric transformation (zoom-decimate, rotate, mirror, shear, warp, perspective transform, affine transform

The Intel software covers wide range of image processing methods applicable in remote sensing and other branches. The largest user group can be found among digital photographs users.

Image Analyzer 1.27. [6] is a freeware for Windows 98/ME/2000/XP/Vista. Advanced image editing, enhancement and analysis software. The program contains both most image enhancement features found in conventional image editors plus a number of advanced features not even available in professional photo suites as:

- Automatic brightness, contrast, gamma and saturation adjustment

- Build-in conventional and adaptive filters for noise reduction, edge extraction etc.

- Retouch tools

- Deconvolution for out-of-focus and motion blur compensation (see below)

- Easy red-eye removal

- User specified filters in spatial and frequency domain

- Resize, rotate, crop and warping of images

- Scanner, camera and printer support

- File format support: Read/write BMP, ICO, CUR, WMF, EMF, PNG, MNG, GIF, PCX, JPEG and JPEG 2000 images

- Morphological operations

- Color model conversion: RGB, CMY, HSI, Lab, YCbCr, YIQ and PCA

- Distance, Fourier and discrete cosine transformation

- Math expression module for creating and transforming images and advanced "pocket" calculator with equation solver

- Plugin system for adding more specialized features. See below for available plugins

\section{Conclusion}

There are more viewers belonging to the software group. Viewers are tools for ready data and users with low level of demands and are prepared for large society of users and created by commercial GIS/remote sensing companies. They are in fact useless for most processing of advanced users and students. The software packages focused on certain tasks are useful for solution of individual problems which cannot be processed in other software on one side or for solution of stand alone tasks. Their application for education of GIS or remote sensing as a whole is not suitable. The university education of GIS and remote sensing can be based on complete software like GRASS and others. The open software allows to use existing modules 
on one side, and are a place for development of new tools on the other side. Therefore, whenever the education is focused on advanced experienced students, these software packages are the best solution for education.

\section{References}

All web pages cited on 18 September 2007

1. http://52north.org/index.php?option=com_projects\&task=showProject\&id=30

2. http://cobweb.ecn.purdue.edu/ ${ }^{\text {biehl/MultiSpec/ }}$

3. http://enterprise.lr.tudelft.nl/doris/

4. http://fmaps.sourceforge.net/

5. http://grass.itc.it/

6. http://meesoft.logicnet.dk/Analyzer/

7. http://opensourcegis.org/

8. http://proj.maptools.org/

9. http://www.esri.com/software/arcexplorer/about/overview.html

10. http://www.fas.org/irp/imint/docs/rst/Front/tofc.html

11. http://www.gdal.org/index.html

12. http://www.intel.com/technology/computing/opencv/overview.htm

13. http://www.intergraph.com/gviewer/Key_Features.asp

14. http://www.ossim.org/OSSIM/OSSIMHome.html

15. http://www.remotesensing.org/Home.html

16. http://www.remotesensing.org/geotiff/geotiff.html

17. http://www.vterrain.org/

The paper was prepared in the framework of the project of the Czech Grant Agency GA ČR 205/06/1037 Application of Geoinformation Technologies for Improvement of Rainfall-Runoff Relationships 
